\title{
Intraperitoneal aerosolization of bupivacaine is a safe and effective method in controlling postoperative pain in laparoscopic Roux-en-Y gastric bypass
}

\author{
Nawar A Alkhamesi ${ }^{1}$ \\ James M Kane, Jr ${ }^{2}$ \\ Paul J Guske ${ }^{2}$ \\ Jonathan W Wallace ${ }^{2}$ \\ Peter C Rantis, Jr ${ }^{2}$ \\ 'Department of Biosurgery \\ and Technology, Imperial College \\ London, UK; ${ }^{2}$ Department of Surgery, \\ Alexian Brothers Hospital Network, \\ Chicago, USA
}

Introduction: Obesity is a worldwide problem and has grown in severity in the last few decades making bariatric surgery and, in particular, laparoscopic banding and Roux-en-Y gastric bypass efficacious and cost-effective procedures. The laparoscopic approach has been shown to offer significant healthcare benefits, of particular interests are reports of decreased postoperative pain resulting in a shorter hospital stay and an earlier return to normal activity. However, many patients still experience significant pain, including shoulder tip pain, that require strong analgesia including opiates during their early recovery period. The aims of this study were to establish the safe use of the aerosolization technique in bariatric surgery and to investigate the possible benefits in reducing postoperative pain.

Methods: In this study, fifty patients undergoing laparoscopic gastric bypass were recruited and divided into two groups; control $(n=25)$ and therapeutic $(n=25)$. The control group received intraperitoneal aerosolization of $10 \mathrm{~mL}$ of $0.9 \%$ normal saline while the therapeutic group received $10 \mathrm{~mL}$ of $0.5 \%$ bupivacaine. All the patients had standard preoperative, intraoperative, and postoperative care. Pain scores were carried out by the nursing staff in recovery and $6 \mathrm{~h}$, $12 \mathrm{~h}$ and $24 \mathrm{~h}$ postoperatively using a standard $0-10$ pain scoring scale. In addition, opiate consumption via patient-controlled analgesia (PCA) was recorded.

Results: Aerosolized bupivacaine reduced postoperative pain in comparison to normal saline $(\mathrm{p}<0.05)$. However, PCA usage showed no statistically significant change from the control group.

Conclusion: The aims of this study were achieved and we were able to establish the safe use of the aerosolization technique in bariatric surgery and its benefits in reducing postoperative pain.

Keywords: laparoscopy, aerosolization, Roux-en-Y gastric bypass, bupivacaine, local anesthetic, intraperitoneal therapeutics

\section{Introduction}

Obesity is a worldwide problem and has grown in severity in the last few decades (Elder and Wolfe 2007). This disease will have a significant impression on patients' health and the economy making bariatric surgery and in particular laparoscopic banding (LAGB) and Roux-en-Y gastric bypass (LRYGB) efficacious and cost-effective procedures (Frezza et al 2007; Hawkins et al 2007). The laparoscopic approach has been shown to offer significant health care benefits, of particular interest are reports of decreased postoperative pain resulting in a shorter hospital stay and an earlier return to normal activity. However, many patients still experience significant pain, including shoulder tip pain, that require strong analgesia including opiate during their early recovery period (Dixon et al 2005; Madan et al 2005). 
Intraperitoneal anesthetics have been studied in LRYGB and the results showed a noticeable reduction in narcotic use with no clear effect on postoperative pain (Symons et al 2007). This finding emphasizes the fact that post-laparoscopic pain is a multifactorial phenomenon and requires a global approach. The author (NA Alkhamesi) has been involved in developing an aerosolization system that utilizes the unique laparoscopic environment, a closed and pressurized system, to deliver intraperitoneal medications in an aerosol form, which will guarantee the uniform coverage of the entire peritoneal cavity in order to achieve maximum therapeutic benefits (Alkhamesi et al 2005). This technology has been shown to be successful in the treatment of post-laparoscopic pain following laparoscopic cholecystectomy using an aerosolized bupivacaine (Alkhamesi et al 2007).

The aims of this study were to establish the safe use of the aerosolization technique in bariatric surgery and to investigate the possible benefits in reducing postoperative pain.

\section{Material and method}

This is a prospective randomized double-blinded clinical trial that was approved by the Institutional Review Board and was carried out at Alexian Brothers Hospital Network, Chicago, USA. All the patients gave informed consent and were provided with an information booklet, which described the study in detail along with the possible risk factors.

\section{Patient selection}

We recruited 50 patients on a voluntary basis who underwent elective laparoscopic Roux-en-Y gastric bypass (LRYGB) at the Alexian Brothers Hospital, Chicago, USA The inclusion criteria included patients between the age of 18-65 years, and American Society of Anesthesiology (ASA) scores I, II, and III. We excluded patients below the age of 18 and those with known allergies to bupivacaine or any other drugs used during anesthesia or in the postoperative pain management protocol. We also excluded patients with ASA IV, patients with history of drug abuse, and those who used narcotic drugs within 30 days of their procedure.

\section{Study design}

All the patients, junior medical staff and the nursing staff in recovery and the wards were blinded to the study. The operating surgeons and the anesthetist were aware of the study protocol, but were blinded to contents of the aerosol or the injected solutions. The 50 patients were randomly divided into 2 groups of twenty-five; the Control group (received $10 \mathrm{~mL}$ of aerosolized intraperitoneal $0.9 \%$ normal saline), and the Treatment group (received $10 \mathrm{~mL}$, equivalent to $50 \mathrm{mg}$ of aerosolized $0.5 \%$ plain bupivacaine).

The two groups had the same anaesthetic protocol. Pre-operatively patients received fentanyl 50-100 $\mu \mathrm{g} / \mathrm{kg}$ and midazolam $0.07 \mathrm{mg} / \mathrm{kg}$ intravenously. Anesthetic induction was achieved by using $1 \mu \mathrm{g} / \mathrm{kg}$ fentanyl and propofol 2-3 mg/kg. Perioperatively, the patients received a mixture of air and oxygen with sevoflurane to maintain anesthesia, in addition, pre-incision $50 \mu \mathrm{g} / \mathrm{kg}$ of morphine was given intravenously. Muscle relaxation was achieved using $0.5 \mathrm{mg} / \mathrm{kg}$ rocuronium.

Experienced laparoscopic surgeons (JK, PG, JW, and PR), using the same surgical technique standardized by the Department of Surgery at the Alexian Brothers Hospital, performed the operations. A classic 6-ports procedure was utilized to perform the operation. Pneumoperitoneum was achieved using nonhumidified $\mathrm{CO}_{2}$ at a pressure of $15 \mathrm{mmHg}$. The medial left upper quadrant trocar site was dilated during the procedure to facilitate the introduction of a size 21 circular stapler (Ethicon, USA). This site was also used to insert the intraperitoneal drain. At the end of the procedure and before ports withdrawal, the intraperitoneal therapeutic was introduced followed by wound closure and the injection of $30 \mathrm{~mL}$ of $0.5 \%$ bupivacaine with epinephrine (150 mg of bupivacaine) into the 6 wounds in all the participant patients. The dose of bupivacaine was calculated at $2 \mathrm{mg} / \mathrm{Kg}$ body weight and did not exceed $210 \mathrm{mg}$ in total for each patient in line with the recommended dosage to avoid any adverse effects.

Postoperative pain management included giving all patients a patient-controlled analgesia (PCA) containing dilaudid or morphine.

The pain was scored by trained nursing staff in recovery, $6 \mathrm{~h}, 12 \mathrm{~h}$, and $24 \mathrm{~h}$ postoperatively using a simple $0-10$ scale where 0 is no pain while 10 is the worst pain experienced by the patients.

\section{Intraperitoneal aerosolization technique}

The local anesthetic and the normal saline were delivered intraoperatively before ports withdrawal and wound closure using the intraperitoneal aerosolization technique developed previously by the author, NA Alkhamesi (Alkhamesi et al $2005,2007)$. In brief, it is a syringe drive integrated with a gas insufflator and uses a specially designed multi-lumen catheter that can be introduced into the abdomen through any port using a special introducer to prevent gas leakage (Figure 1). This system enables the operator to deliver the 


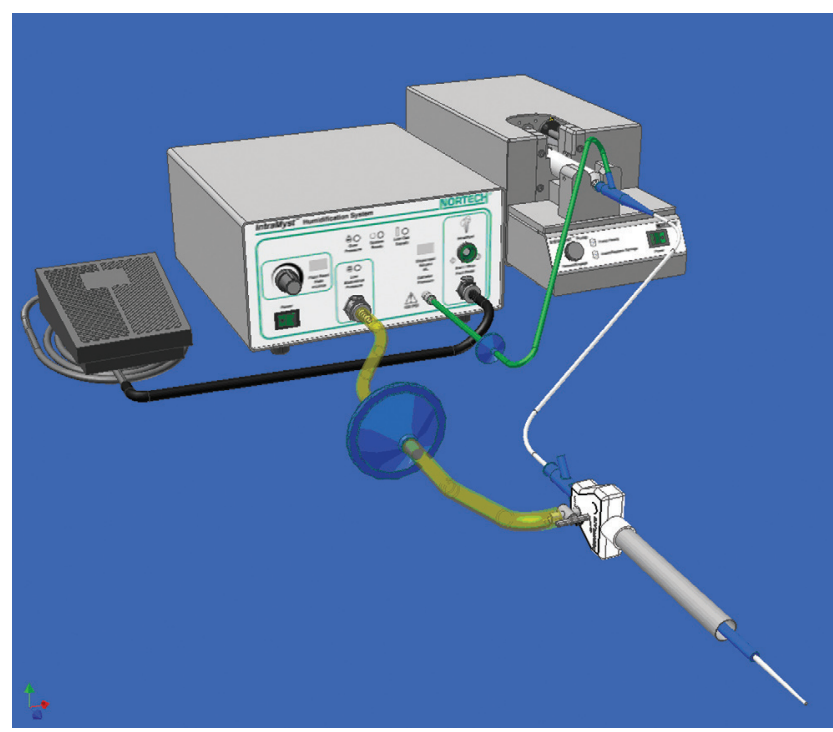

Figure I Intraperitoneal aerosolization device. The syringe drive on the right is connected to the insufflator using a specially designed single use catheter (Green and White).

desired therapeutic dose while employing the smallest possible volume.

In this study, the surgeons were able to aerosolize either $10 \mathrm{~mL}$ of $0.5 \%$ bupivacaine or $10 \mathrm{~mL}$ of $0.9 \%$ normal saline at a controlled speed of $3.5 \mathrm{~mL} / \mathrm{min}$. This delivery method generated a "foggy" environment to ensure that the intraperitoneal space was covered completely.

\section{Statistical analysis}

Analysis of variance (ANOVA) was used to compare multiple group means. Differences between individual groups were examined using post hoc analysis (Tukey-Kramer test). SPSS $^{\mathrm{TM}} 11.5$ for Windows ${ }^{\mathrm{TM}}$ (SPSS Inc., Chicago, IL, USA) was utilized to perform all statistics. All data are presented as the mean \pm standard error of the mean (SEM). A p value of less than 0.05 was taken to be significant.

\section{Results}

In this study, 50 patients were recruited with mean age of 43 years (range, 21-65 years). There were 35 females and 15 males with an average body mass index (BMI) of 51.95 (range, 34.1-69.8). They were divided into two groups. The control group $(\mathrm{N}=25)$ had 17 females and 8 males with mean age of 46.3 years (range, 26-65 years) and the average BMI was 50.2 (range, 35.9-69.8). The therapeutic group $(\mathrm{N}=25)$ consisted of 18 females and 7 males with mean age of 41.2 years (range, 21-65 years). and the average BMI was 47.1 (range, 34.1-68.9).

The aerosolization of $0.5 \%$ bupivacaine reduced postoperative pain including shoulder tip pain in comparison with the control group, $\mathrm{p}=0.01$, maximum reduction was in the recovery period and continued at all other given time (Figure 2). This reduction in pain severity was matched by a noticeable decrease in PCA usage in the therapeutic

\section{Average pain scores}

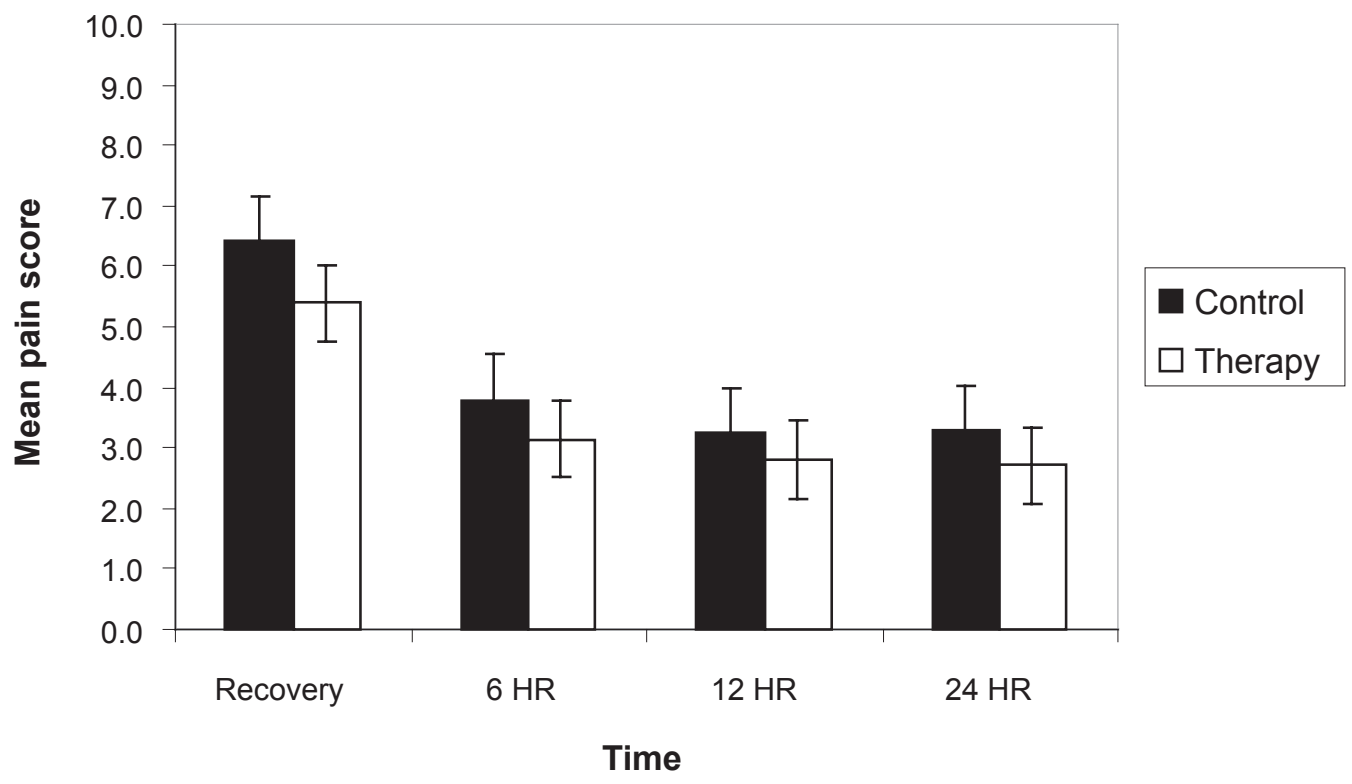

Figure 2 The aerosolisation of $0.5 \%$ bupivacaine reduced postoperative pain in comparison with control. Trained nursing staff in recovery and the ward using a $0-10$ scale recorded the pain severity. 
groups; however, it was not statistically significance, $\mathrm{p}=0.52$ (Figure 3).

All the patients were discharged home as planned and none of them suffered any side effects that might be associated with the aerosolization device or the bupivacaine used.

\section{Discussion}

Despite all the benefits that have come with the introduction of minimal invasive surgery, postoperative pain and, in particular, shoulder pain remains an issue.

Many studies have tried to address the notion of postlaparoscopic pain; they exploited many methods including injecting the port site with local anesthetic, intraperitonal administration of normal saline or bupivavcaine (Liu et al 1993; Alexander et al 1996; Michaloliakou et al 1996; Cunniffe et al 1998; Bar-Dayan et al 2004), and the use of warm and humidified gas (Korell et al 1996; Huntington and LeMaster 1997; Mouton et al 1999a, 2001). No techniques achieved a clear benefit and the results were contradicting (Joris et al 1995; Deans et al 1998; Mouton et al 1999b; Ghezzi et al 2005).

Alkhamesi and colleagues have successfully used the aerosolazation technique in the management of postoperative pain following laparoscopic cholecystectomy (Alkhamesi et al 2007). They showed that bupivacaine aerosol significantly reduced pain in comparison to control and to the administration of local anesthetic in to the gall bladder bed.
In this study, we utilized a novel aerosolization system that has been proven to be successful in delivering intraperitoneal local anesthetics to treat postoperative pain. Patients in the therapeutic group had less pain at all measured time points compared to the control group and used their PCA less. Although the statistics did not show any significant difference in the PCA usage, we believe that, this was because the patients were instructed, at their pre-operative consultation, to use their PCA regularly even if they had no pain. This fact might have a major effect on our study outcome and the investigators were not aware of it until they started analyzing the data.

Moreover, this study demonstrated that the aerosolization technique is safe in bariatric patients and can be used to deliver intraperitoneal therapeutics during laparoscopic procedures.

In conclusion, the aims of this study were achieved and we were able to establish the safe use of the aerosolization technique in bariatric surgery and identify its potential benefits for reducing postoperative pain. The authors believe that a better organized trial with higher numbers in each group is required to confirm the status of this method of pain control in laparoscopic bariatric operations in order for it to become the standard technique. We also believe that this method of pain management can be used in other laparoscopic operations such as laparoscopic ventral hernia repair and laparoscopic colorectal resection.

\section{PCA dosage (mgs)}

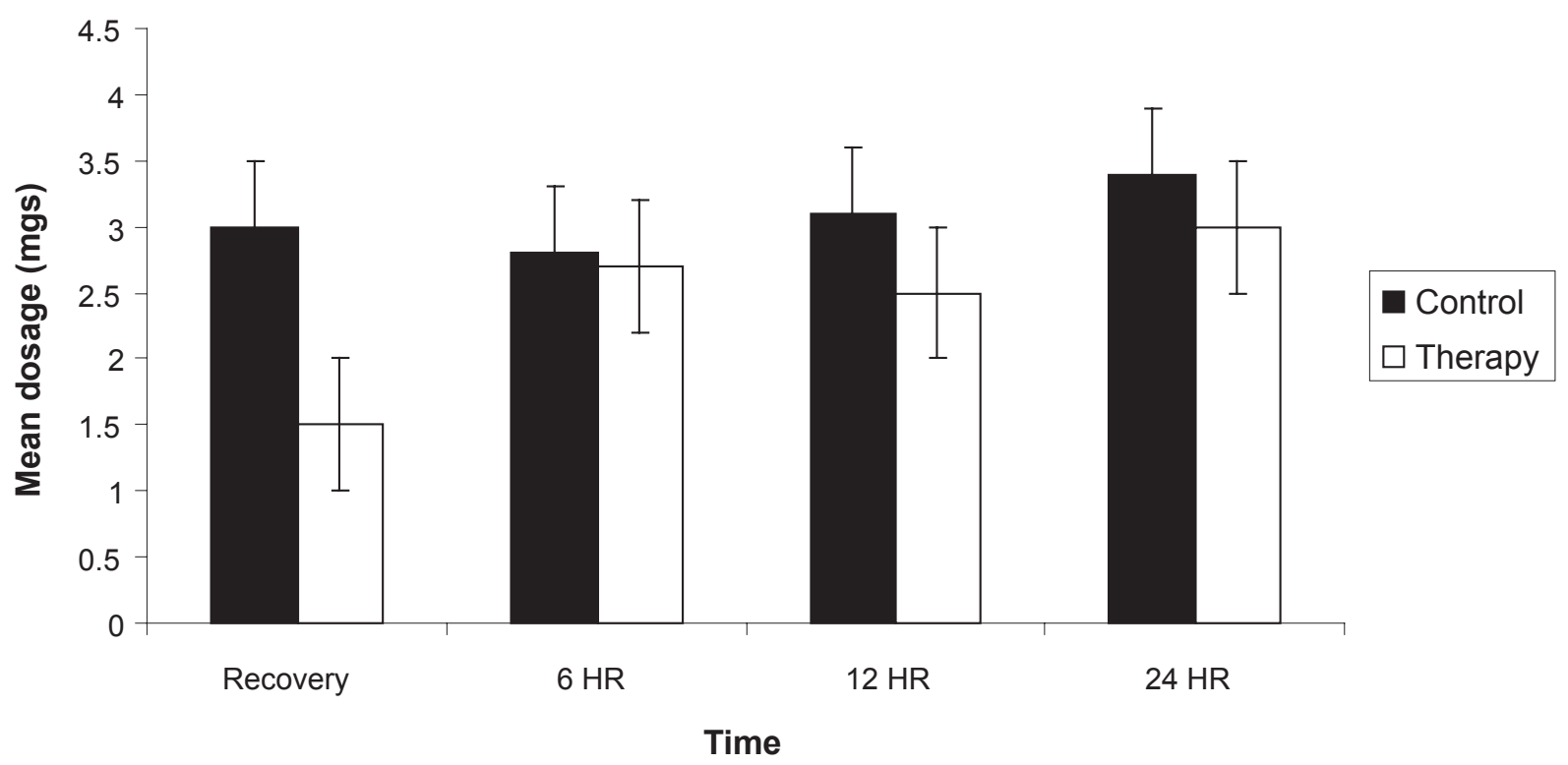

Figure 3 The use of PCA was reduced in the therapeutic group in comparison with control. However, it did not reach statistical significance in all measured time points. 


\section{Disclosure}

The authors report no conflicts of interest in this work.

\section{References}

Alexander DJ, Ngoi SS, Lee L, et al. 1996. Randomized trial of periportal peritoneal bupivacaine for pain relief after laparoscopic cholecystectomy. Br J Surg, 83:1223-5.

Alkhamesi NA, Peck DH, Lomax D, et al. 2007. Intraperitoneal aerosolization of bupivacaine reduces postoperative pain in laparoscopic surgery: a randomized prospective controlled double-blinded clinical trial. Surg Endosc, 21:602-6.

Alkhamesi NA, Ridgway PF, Ramwell A, et al. 2005. Peritoneal nebulizer: a novel technique for delivering intraperitoneal therapeutics in laparoscopic surgery to prevent locoregional recurrence. Surg Endosc, 19:1142-6.

Bar-Dayan A, Natour M, Bar-Zakai B, et al. 2004. Preperitoneal bupivacaine attenuates pain following laparoscopic inguinal hernia repair. Surg Endosc, 18:1079-81.

Cunniffe MG, McAnena OJ, Dar MA, et al. 1998. A prospective randomized trial of intraoperative bupivacaine irrigation for management of shoulder-tip pain following laparoscopy. Am J Surg, 176:258-61.

Deans GT, Wilson MS, Brough WA. 1998. Controlled trial of preperitoneal local anaesthetic for reducing pain following laparoscopic hernia repair. Br J Surg, 85:1013-4.

Dixon JB, Reuben Y, Halket C, et al. 2005. Shoulder pain is a common problem following laparoscopic adjustable gastric band surgery. Obes Surg, 15:1111-7.

Elder KA, Wolfe BM. 2007. Bariatric surgery: a review of procedures and outcomes. Gastroenterology, 132:2253-71.

Frezza EE, Wachtel MS, Ewing BT. 2007. Bariatric surgery costs and implications for hospital margins: Comparing laparoscopic gastric bypass and laparoscopic gastric banding. Surg Laparosc Endosc Percutan Tech, 17:239-44.
Ghezzi F, Cromi A, Bergamini V, et al. 2005. Preemptive port site local anesthesia in gynecologic laparoscopy: a randomized, controlled trial. J Minim Invasive Gynecol, 12:210-5.

Hawkins SC, Osborne A, Finlay IG, et al. 2007. Paid work increases and state benefit claims decrease after bariatric surgery. Obes Surg, 17:434-7.

Huntington TR, LeMaster CB. 1997. Laparoscopic hypothermia: heat loss from insufflation gas flow. Surg Laparosc Endosc, 7:153-5.

Joris J, Thiry E, Paris P, et al. 1995. Pain after laparoscopic cholecystectomy: characteristics and effect of intraperitoneal bupivacaine. Anesth Analg, 81:379-84.

Korell M, Schmaus F, Strowitzki T, et al. 1996. Pain intensity following laparoscopy. Surg Laparosc Endosc, 6:375-9.

Liu J, Ding Y, White PF, et al. 1993. Effects of ketorolac on postoperative analgesia and ventilatory function after laparoscopic cholecystectomy. Anesth Analg, 76:1061-6.

Madan AK, Ternovits CA, Speck KE, et al. 2005. Inpatient pain medication requirements after laparoscopic gastric bypass. Obes Surg, 15:778-81.

Michaloliakou C, Chung F, Sharma S. 1996. Preoperative multimodal analgesia facilitates recovery after ambulatory laparoscopic cholecystectomy. Anesth Analg, 82:44-51.

Mouton WG, Bessell JR, Millard SH, et al. 1999a. A randomized controlled trial assessing the benefit of humidified insufflation gas during laparoscopic surgery. Surg Endosc, 13:106-8.

Mouton WG, Bessell JR, Otten KT, et al. 1999b. Pain after laparoscopy. Surg Endosc, 13:445-8.

Mouton WG, Naef M, Bessell JR, et al. 2001. A randomized controlled trial to determine the effect of humidified carbon dioxide (CO2) insufflation on postoperative pain following thoracoscopic procedures. Surg Endosc, 15:579-81.

Symons JL, Kemmeter PR, Davis AT, et al. 2007. A double-blinded, prospective randomized controlled trial of intraperitoneal bupivacaine in laparoscopic Roux-en-Y gastric bypass. J Am Coll Surg, 204:392-8. 
